\title{
Exploring quantitative group-wise differentiation of Alzheimer's disease and behavioural variant frontotemporal dementia using tract-specific microstructural white matter and functional connectivity measures at multiple time points
}

\author{
R. Meijboom ${ }^{1,2} \cdot$ R. M. E. Steketee ${ }^{1}$ - L. S. Ham $^{1}$ - D. Mantini ${ }^{3,4} \cdot$ E. E. Bron ${ }^{5} \cdot$ A. van der Lugt ${ }^{1} \cdot$ J. C. van Swieten $^{6} \cdot$ \\ M. Smits ${ }^{1}$
}

Received: 5 September 2018 / Revised: 7 January 2019 / Accepted: 1 February 2019 / Published online: 11 March 2019

(C) The Author(s) 2019

\begin{abstract}
Objectives This study explored group-wise quantitative measures of tract-specific white matter (WM) microstructure and functional default mode network (DMN) connectivity to establish an initial indication of their clinical applicability for early-stage and follow-up differential diagnosis of Alzheimer's disease (AD) and behavioural variant frontotemporal dementia (bvFTD).

Methods Eleven AD and 12 bvFTD early-stage patients and 18 controls underwent diffusion tensor imaging and resting state functional magnetic resonance imaging at 3 T. All AD and 6 bvFTD patients underwent the same protocol at 1-year follow-up. Functional connectivity measures of DMN and WM tract-specific diffusivity measures were determined for all groups. Exploratory analyses were performed to compare all measures between the three groups at baseline and between patients at follow-up. Additionally, the difference between baseline and follow-up diffusivity measures in AD and bvFTD patients was compared.

Results Functional connectivity of the DMN was not different between groups at baseline and at follow-up. Diffusion abnormalities were observed widely in bvFTD and regionally in the hippocampal cingulum in AD. The extent of the differences between bvFTD and AD was diminished at follow-up, yet abnormalities were still more pronounced in bvFTD. The rate of change was similar in bvFTD and AD.

Conclusions This study provides a tentative indication that quantitative tract-specific microstructural WM abnormalities, but not quantitative functional connectivity of the DMN, may aid early-stage and follow-up differential diagnosis of bvFTD and AD. Specifically, pronounced microstructural changes in anterior WM tracts may characterise bvFTD, whereas microstructural abnormalities of the hippocampal cingulum may characterise AD.

Key Points

- The clinical applicability of quantitative brain imaging measures for early-stage and follow-up differential diagnosis of dementia subtypes was explored using a group-wise approach.
\end{abstract}

Electronic supplementary material The online version of this article (https://doi.org/10.1007/s00330-019-06061-7) contains supplementary material, which is available to authorized users.

M. Smits

marion.smits@erasmusmc.nl

1 Department of Radiology and Nuclear Medicine, Erasmus MC University Medical Centre Rotterdam, Rotterdam, The Netherlands

2 Centre for Clinical Brain Sciences, University of Edinburgh, Edinburgh, United Kingdom

3 Research Center for Motor Control and Neuroplasticity, KU Leuven, Leuven, Belgium
4 Functional Neuroimaging Laboratory, IRCCS San Camillo Hospital Foundation, Lido, Italy

5 Biomedical Imaging Group Rotterdam - Departments of Medical Informatics and Radiology, Erasmus MC - University Medical Centre Rotterdam, Rotterdam, The Netherlands

6 Department of Neurology, Erasmus MC - University Medical Centre Rotterdam, Rotterdam, The Netherlands 
- Quantitative tract-specific microstructural white matter abnormalities, but not quantitative functional connectivity of the default mode network, may aid early-stage and follow-up differential diagnosis of behavioural variant frontotemporal dementia and Alzheimer's disease.

- Pronounced microstructural white matter (WM) changes in anterior WM tracts characterise behavioural variant frontotemporal dementia, whereas microstructural WM abnormalities of the hippocampal cingulum in the absence of other WM changes characterise Alzheimer's disease.

Keywords Alzheimer disease $\cdot$ Frontotemporal dementia $\cdot$ Functional MRI $\cdot$ Diffusion tensor imaging $\cdot$ Longitudinal studies

$\begin{array}{ll}\text { Abbreviations } \\ \text { AD } & \text { Alzheimer's disease } \\ \text { ATR } & \text { Anterior thalamic radiation } \\ \text { AxD } & \text { Axial diffusivity } \\ \text { BOLD } & \text { Blood-oxygenation-level dependent } \\ \text { bvFTD } & \text { Behavioural variant frontotemporal dementia } \\ \text { CGC } & \text { Cingulate cingulum } \\ \text { CGH } & \text { Hippocampal cingulum } \\ \text { DMN } & \text { Default mode network } \\ \text { DTI } & \text { Diffusion tensor imaging } \\ \text { EPI } & \text { Echo planar imaging } \\ \text { FA } & \text { Fractional anisotropy } \\ \text { FDR } & \text { False discovery rate } \\ \text { FMA } & \text { Forceps major } \\ \text { FMI } & \text { Forceps minor } \\ \text { FSL } & \text { FMRIB Software Library } \\ \text { FSPGR } & \text { Fast spoiled gradient echo } \\ \text { GM } & \text { Grey matter } \\ \text { ICV } & \text { Intracranial volume } \\ \text { IFOF } & \text { Inferior fronto-occipital fasciculus } \\ \text { ILF } & \text { Inferior longitudinal fasciculus } \\ \text { IR } & \text { Inversion recovery } \\ \text { MD } & \text { Mean diffusivity } \\ \text { MMSE } & \text { Mini-Mental State Examination } \\ \text { MNI } & \text { Montreal Neurological Institute } \\ \text { MRI } & \text { Magnetic resonance imaging } \\ \text { RD } & \text { Radial diffusivity } \\ \text { ROIs } & \text { Regions of interest } \\ \text { rs-fMRI } & \text { Resting state functional magnetic resonance } \\ & \text { imaging } \\ \text { SLF } & \text { Superior longitudinal fasciculus } \\ \text { SPM } & \text { Statistical Parametric Mapping } \\ \text { T1w } & \text { T1-weighted } \\ \text { UF } & \text { Uncinate fasciculus } \\ \text { WM } & \text { White matter } \\ & \end{array}$

\section{Introduction}

Presenile dementia is a dementia with an onset before the age of 65 years. The two most common underlying disorders are Alzheimer's disease (AD) and behavioural variant frontotemporal dementia (bvFTD) [1]. AD is characterised by an episodic memory disturbance for recently learned as well as for learning new material, together with at least one other cognitive disturbance [2]. In contrast, bvFTD is mainly characterised by behavioural problems such as disinhibition, apathy and loss of empathy [3]. In later stages of $\mathrm{AD}$ and bvFTD, predominance of cognitive impairment in $\mathrm{AD}$ and social/executive impairment in bvFTD $[4,5]$ aids differential diagnosis. However, differential diagnosis can be difficult in early stages of AD and bvFTD, as symptoms may still be mild and unspecific. BvFTD patients may present with memory deficits $[6,7]$ and $A D$ patients with changes in social behaviour or executive functioning $[5,7,8]$. Magnetic resonance imaging (MRI) supports diagnosis, but in early disease stages, conventional (structural) MRI may still appear normal or show diffuse brain abnormalities unspecific for a dementia subtype [9-11]. More advanced MRI techniques, such as diffusion tensor imaging (DTI) and resting state functional MRI (rs-fMRI), may aid differential diagnosis by detecting more subtle abnormalities that remain unrevealed using structural MRI [12].

DTI is used to assess white matter (WM) microstructure of the brain. Previous studies observed more pronounced microstructural WM abnormalities in bvFTD than in AD $[13,14]$ and suggested an anterior-posterior division of WM abnormalities in bvFTD and AD. Microstructural WM abnormalities are observed in the anterior brain regions in bvFTD, such as the cingulate cingulum (CGH), forceps minor (FMI) and uncinate fasciculus (UF), whereas microstructural WM changes in $\mathrm{AD}$ are localised in more posterior brain regions, such as the forceps major (FMA) and the hippocampal cingulum (CGH) [15-17]. Rs-fMRI is used to assess functional connectivity between grey matter (GM) regions that together form functional brain networks. A widely studied network is the default mode network (DMN), known to be affected in both AD and bvFTD [18]. Previous research has shown DMN differences between $\mathrm{AD}$ and bvFTD, specifically decreased DMN connectivity in $\mathrm{AD}$ and increased DMN connectivity in bvFTD - mostly in the posterior DMN.

Clinical diagnosis may especially benefit from objective quantitative measures derived from DTI and rs-fMRI in differentiating subtypes of dementia patients, and patients 
from healthy persons, using group-specific reference values. In this study, we explored group-wise quantitative measures of tract-specific WM microstructure and functional connectivity of the DMN in a small patient population, to provide an initial indication of their diagnostic utility for early-stage and over time differentiation of $\mathrm{AD}$ and bvFTD.

\section{Methods}

\section{Participants}

Patients with a suspected diagnosis of early AD or bvFTD were recruited soon after their initial visits to the Alzheimer Centre Rotterdam. Suspected AD or bvFTD diagnosis was established by a multidisciplinary team of neurologists, neuroradiologists, nuclear radiologists, geriatricians and neuropsychologists. Diagnostic criteria included patient complaints, medical history, neurological examination, radiological assessment and full cognitive assessment that were overall suggestive of AD or bvFTD and in line with the established diagnostic criteria for AD [2] and bvFTD [3]. Genetic testing was performed only in case of a positive family history for dementia. Six bvFTD patients included in this study had a genetic mutation (5 MAPT, 1 C9ORF72).

Inclusion criteria for this study were an age between 40 and 70 years; suspected diagnosis of early AD [2] or bvFTD [3]; a Mini-Mental State Examination [19] (MMSE) score of $\geq 20$. Exclusion criteria were contraindications for MRI; an expected loss to follow-up within one year; other neurological disorders; a different cause of dementia; alternative psychiatric diagnosis; past or current substance abuse. Diagnosis of either AD or bvFTD was confirmed after at least one year follow-up. Patients underwent the Mini-Mental State Examination (MMSE) as part of their routine clinical diagnostic work-up. Healthy controls, matched for age and gender, and without neurological or psychiatric history, were recruited through advertisement. Controls underwent neuropsychological testing and the MMSE as part of this study to rule out cognitive impairment. The study was approved by the local medical ethics committee. All participants gave written informed consent.

\section{Image acquisition}

MRI was performed on a 3 T Discovery MR750 system (GE Healthcare). See Table 1 for acquisition parameters. Patients underwent identical MRI protocols at baseline (T0) and at 1-year follow-up (T1). Controls underwent MRI at T0 only. For anatomical reference, a highresolution three-dimensional (3D) inversion recovery (IR) fast spoiled gradient echo (FSPGR) T1-weighted (T1w)
Table 1 Acquisition parameters

\begin{tabular}{llll}
\hline & T1w & DTI & fMRI \\
\hline FOV (mm) & 240 & 240 & 240 \\
TE (ms) & 3.06 & $84.5^{*}$ & 30 \\
TR (ms) & 7904 & 7930 & 3000 \\
ASSET factor & 2 & 2 & 2 \\
Flip angle & $12^{\circ}$ & $90^{\circ}$ & $90^{\circ}$ \\
Acquisition matrix & $240 \times 240$ & $128 \times 128$ & $96 \times 96$ \\
Slice thickness (mm) & 1 & 2.5 & 3 \\
Volumes (slices per volume) & $1(176)$ & $28(59)$ & $200(44)$ \\
Duration (min) & 4.41 & 3.50 & 10.00 \\
Diffusion-weighted directions & $\mathrm{n} / \mathrm{a}$ & 25 & $\mathrm{n} / \mathrm{a}$ \\
Non-diffusion weighted images & $\mathrm{n} / \mathrm{a}$ & 3 & $\mathrm{n} / \mathrm{a}$ \\
Maximum $b$ value (s/ mm ${ }^{2}$ ) & $\mathrm{n} / \mathrm{a}$ & 1000 & $\mathrm{n} / \mathrm{a}$ \\
TI (ms) & 450 & $\mathrm{n} / \mathrm{a}$ & $\mathrm{n} / \mathrm{a}$ \\
\hline
\end{tabular}

T1w, T1-weighted; DTI, diffusion tensor imaging; $f M R I$, functional magnetic resonance imaging; $F O V$, field of view; $T E$, echo time; $T R$, repetition time; $A S S E T$, array spatial sensitivity encoding technique; $T I$, inversion time

*TE for DTI was set to minimum. This number represents the average TE. The range of TE was 81.9-90.8 ms

image was acquired. DTI scans were acquired with spinecho echo planar imaging (EPI) and rs-fMRI scans with gradient echo EPI. For rs-fMRI, participants were instructed to think of nothing in particular, to focus on a fixation cross and to remain awake.

\section{Demographical analysis}

Between-group differences in age were tested using ANOVA. Between-group differences in MMSE score were tested using the Welch-ANOVA and post hoc Games-Howell $t$ tests, due to unequal variance across groups. Gender was compared across groups using the chi-square tests. Analyses were done using IBM SPSS Statistics 21.0 with a significance threshold of $p<0.05$.

\section{GM volume analysis}

GM volumes were calculated according to the methods described in Bron et al (2014) [20]. GM volumes were obtained from the T1w image using the unified tissue segmentation method of Statistical Parametric Mapping (SPM8), after which intracranial volume (ICV) was calculated. Then, GM volume was divided by ICV to correct for brain size. GM volume (\%ICV) was compared for groups at $\mathrm{T} 0$ and at $\mathrm{T} 1 \mathrm{using}$ ANOVA and the post hoc Bonferroni tests. 


\section{Microstructural WM analysis}

WM tracts known to be associated with cognitive functions were selected for tractography: anterior thalamic radiation (ATR) [21], cingulum (CGH and CGC) [22], FMA [21], FMI [21, 23], inferior fronto-occipital fasciculus (IFOF) $[24,25]$, inferior longitudinal fasciculus (ILF) [24, 25], superior longitudinal fasciculus (SLF) $[26,27]$ and UF $[22,23,25]$.

Tracts were generated using automated probabilistic tractography (AutoPtx) as implemented in FMRIB Software Library (FSL5) [28]. Median fractional anisotropy (FA), mean diffusivity (MD), radial diffusivity (RD) and axial diffusivity (AxD) were established for each tract. The quality of WM tracts was visually assessed. See supplement $\$ 1$ for a full description.

The rate of change (T1-T0) was established for each diffusion measure for each tract. Then, diffusion measures at T0 and $\mathrm{T} 1$ and the rates of change were compared between groups using ANOVA and the post hoc Bonferroni $t$ tests, unless an age effect was present. Age effects were investigated using linear regression and, if necessary, taken into account using ANCOVA. In case of unequal variances across groups, between-group differences were investigated using the WelchANOVA and post hoc Games-Howell $t$ tests.

\section{Functional connectivity analysis}

Using regions of interest (ROIs) of the Hammers atlas (30 atlases with 83 ROIs; http://brain-development.org/ brain-atlases) [29], GM regions making up the DMN were selected for functional analysis: bilateral medial prefrontal cortex, lateral temporal cortex, inferior parietal lobule, precuneus and posterior cingulate cortex. ROIs were normalised to Montreal Neurological Institute (MNI) space.

Functional and structural data were pre-processed using SPM8 (supplement \$2). This was followed by further preprocessing and analysis using the connectivity toolbox by Mantini [30, 31]. For each ROI, the average blood oxygenation level-dependent (BOLD) signal was calculated. Subsequently, the average BOLD signal of each ROI was correlated with all ROIs separately to assess functional connectivity. A Fisher's $r$-to- $z$ transformation was then applied to allow analysis of between-group functional connectivity differences. For both T0 and T1, functional connectivity between ROIs was established for each group using a random-effect analysis corrected for multiple comparisons (false discovery rate $(\mathrm{FDR})<0.001)$. Between-group differences at $\mathrm{T} 0$ and at T1 were assessed using ANCOVA $(p<0.05)$ with GM volume $(\% \mathrm{ICV})$ as covariate and as post hoc two-sample $t$ tests $(\mathrm{FDR}<0.05)$.

\section{Results}

\section{Baseline (T0)}

\section{Participant characteristics}

Baseline data from 11 AD patients, 12 bvFTD patients ( 9 for rs-fMRI) and 18 controls were used for the analysis (Table 2; see supplement \$3 for exclusions).

Participants did not differ in age $(F(2,38)=0.498$, $p>0.05)$, gender $\left(\chi^{2}(2)=2.288, p>0.05\right)$ or education level $\left(\chi^{2}(4)=3.394, p>0.05\right)$. Education level was unknown for 2 bvFTD patients, 1 AD patient and 1 control. MMSE score was different between groups $(F(2,17.1)=20.213, p<0.001)$ and was lower in both patient groups compared with controls. MMSE score did not differ between AD and bvFTD.

\section{GM volume}

The total GM volume (\% ICV) was significantly lower $(F(2,38)=13.837, p<0.001)$ in bvFTD $(0.30 \% \mathrm{ICV}$, standard deviation (SD) 0.04$)$ compared with both $\mathrm{AD}(0.33 \% \mathrm{ICV}, \mathrm{SD}$ 0.03 ) and controls $(0.36 \% \mathrm{ICV}$, SD 0.03$)$, but not different between $\mathrm{AD}$ and controls.

\section{WM microstructure}

WM tracts were correctly identified in all groups. AD in comparison with controls (Table 3 ) showed higher MD only in the right $\mathrm{CGH}$. BvFTD in comparison with controls (Table 3; Fig. 1) showed lower FA and higher MD, RD and $\mathrm{AxD}$ in the bilateral CGH, IFOF, UF and FMI. Higher MD, RD and AxD in bvFTD compared with controls were additionally observed in the bilateral ATR, ILF and SLF. Further, lower FA and higher MD and RD in bvFTD compared with controls were observed in the bilateral CGC. BvFTD in comparison with AD (Table 3; Fig. 1) showed lower FA and higher MD, $\mathrm{RD}$ and $\mathrm{AxD}$ in the bilateral IFOF and UF and FMI. Higher $\mathrm{MD}, \mathrm{RD}$ and $\mathrm{AxD}$ in bvFTD compared with AD were

Table 2 Demographic characteristics

\begin{tabular}{lrll}
\hline Group & \multicolumn{1}{l}{$N$} & Mean age & Mean MMSE \\
\hline BvFTD & $12(6$ male $)$ & $60.3(7.7)$ & $26.6(2.8)$ \\
BvFTD T1 & $6(3$ male $)$ & $64.0(3.6)$ & - \\
AD & $11(8$ male $)$ & $62.8(5.0)$ & $25.3(2.0)$ \\
AD T1 & $11(8$ male $)$ & $63.3(5.0)$ & - \\
Controls & $18(8$ male $)$ & $59.8(6.7)$ & $29.1(1.0)$ \\
\hline
\end{tabular}

$B v F T D$, behavioural variant frontotemporal dementia; $A D$, Alzheimer's disease; $N$, sample size. Values given as mean (standard deviation). MMSE, Mini-Mental State Examination 
Table 3 Mean FA, MD, RD and AxD for each WM tract, for bvFTD, AD and controls at T0. The numbers shown are multiplied by a factor 1000

\begin{tabular}{|c|c|c|c|c|c|c|c|c|c|c|c|c|c|}
\hline \multirow[t]{2}{*}{ WM tract } & \multirow[t]{2}{*}{$\mathrm{L} / \mathrm{R}$} & \multicolumn{3}{|l|}{ FA } & \multicolumn{3}{|l|}{ MD } & \multicolumn{3}{|l|}{$\mathrm{RD}$} & \multicolumn{3}{|l|}{$\mathrm{AxD}$} \\
\hline & & BvFTD & $\mathrm{AD}$ & Controls & BvFTD & $\mathrm{AD}$ & Controls & BvFTD & $\mathrm{AD}$ & Controls & BvFTD & $\mathrm{AD}$ & Controls \\
\hline ATR & $\mathrm{L}$ & 320.44 & 328.95 & 327.82 & 0.90 & 0.83 & $0.81 *$ & 0.73 & 0.68 & 0.67 & 1.27 & 1.18 & $1.15^{*}$ \\
\hline ATR & $\mathrm{R}$ & 315.64 & 320.27 & 322.81 & 0.93 & 0.83 & 0.82 & 0.76 & 0.69 & 0.68 & 1.31 & 1.17 & $1.16^{*}$ \\
\hline CGC & $\mathrm{L}$ & 392.62 & 417.66 & 438.54 & 0.86 & 0.80 & 0.81 & 0.67 & 0.61 & 0.60 & 1.26 & 1.20 & 1.23 \\
\hline CGC & $\mathrm{R}$ & 355.58 & 384.25 & 403.92 & 0.87 & 0.81 & $0.81 *$ & 0.70 & 0.63 & 0.62 & 1.23 & 1.17 & 1.19 \\
\hline $\mathrm{CGH}$ & $\mathrm{L}$ & 221.51 & 256.26 & 266.75 & 0.97 & 0.88 & $0.84 *$ & 0.85 & 0.76 & $0.72 *$ & 1.27 & 1.19 & $1.13 *$ \\
\hline $\mathrm{CGH}$ & $\mathrm{R}$ & 213.55 & 244.43 & 264.69 & 1.04 & 0.88 & $0.84 *$ & 0.91 & 0.76 & $0.72 *$ & 1.34 & 1.19 & $1.16^{*}$ \\
\hline IFOF & $\mathrm{L}$ & 380.55 & 403.52 & 415.33 & 0.89 & 0.83 & 0.82 & 0.68 & 0.64 & 0.62 & 1.30 & 1.23 & 1.23 \\
\hline IFOF & $\mathrm{R}$ & 380.37 & 402.05 & 425.34 & 0.89 & 0.84 & $0.82 *$ & 0.70 & 0.64 & 0.61 & 1.31 & 1.24 & $1.24 *$ \\
\hline ILF & $\mathrm{L}$ & 373.10 & 383.60 & 388.35 & 0.86 & 0.83 & 0.82 & 0.67 & 0.64 & 0.63 & 1.25 & 1.21 & 1.19 \\
\hline ILF & $\mathrm{R}$ & 382.13 & 389.16 & 403.75 & 0.86 & 0.83 & 0.82 & 0.67 & 0.64 & 0.62 & 1.27 & 1.22 & $1.20 *$ \\
\hline SLF & $\mathrm{L}$ & 310.14 & 327.78 & 330.49 & 0.84 & 0.80 & 0.79 & 0.69 & 0.65 & 0.65 & 1.16 & 1.12 & 1.11 \\
\hline SLF & $\mathrm{R}$ & 309.32 & 325.56 & 330.63 & 0.84 & 0.80 & 0.79 & 0.70 & 0.65 & 0.65 & 1.17 & 1.12 & 1.12 \\
\hline UF & $\mathrm{L}$ & 294.48 & 329.90 & 355.41 & 0.95 & 0.83 & $0.82 *$ & 0.79 & 0.68 & $0.65^{*}$ & 1.29 & 1.17 & $1.17 *$ \\
\hline UF & $\mathrm{R}$ & 282.49 & 323.75 & 338.02 & 0.98 & 0.85 & $0.84 *$ & 0.83 & 0.69 & $0.68^{*}$ & 1.33 & 1.19 & $1.19 *$ \\
\hline FMI & $\mathrm{n} / \mathrm{a}$ & 319.98 & 387.77 & 421.05 & 0.96 & 0.85 & $0.82 *$ & 0.78 & 0.66 & $0.62 *$ & 1.30 & 1.23 & 1.23 \\
\hline FMA & $\mathrm{n} / \mathrm{a}$ & 383.12 & 372.15 & 399.79 & 0.83 & 0.83 & 0.80 & 0.64 & 0.64 & 0.61 & 1.30 & 1.30 & 1.29 \\
\hline
\end{tabular}

Italicised entries $=$ vs other patient group $p<0.05$; Bold entries $=$ vs controls $p<0.05$

$F A$, fractional anisotropy; $M D$, mean diffusivity; $R D$, radial diffusivity; $A x D$, axial diffusivity; $W M$, white matter; $b v F T D$, behavioural variant frontotemporal dementia; $A D$, Alzheimer's disease; $A T R$, anterior thalamic radiation; $C G C$, cingulum (cingulate); $C G H$, cingulum (hippocampal); $I F O F$, inferior fronto-occipital fasciculus; $I L F$, inferior longitudinal fasciculus; $S L F$, superior longitudinal fasciculus; $U F$, uncinate fasciculus; $F M I$, forceps minor; $F M A$, forceps major; $L$, left; $R$, right

*Unequal variance, significance tested with the Welch-ANOVA

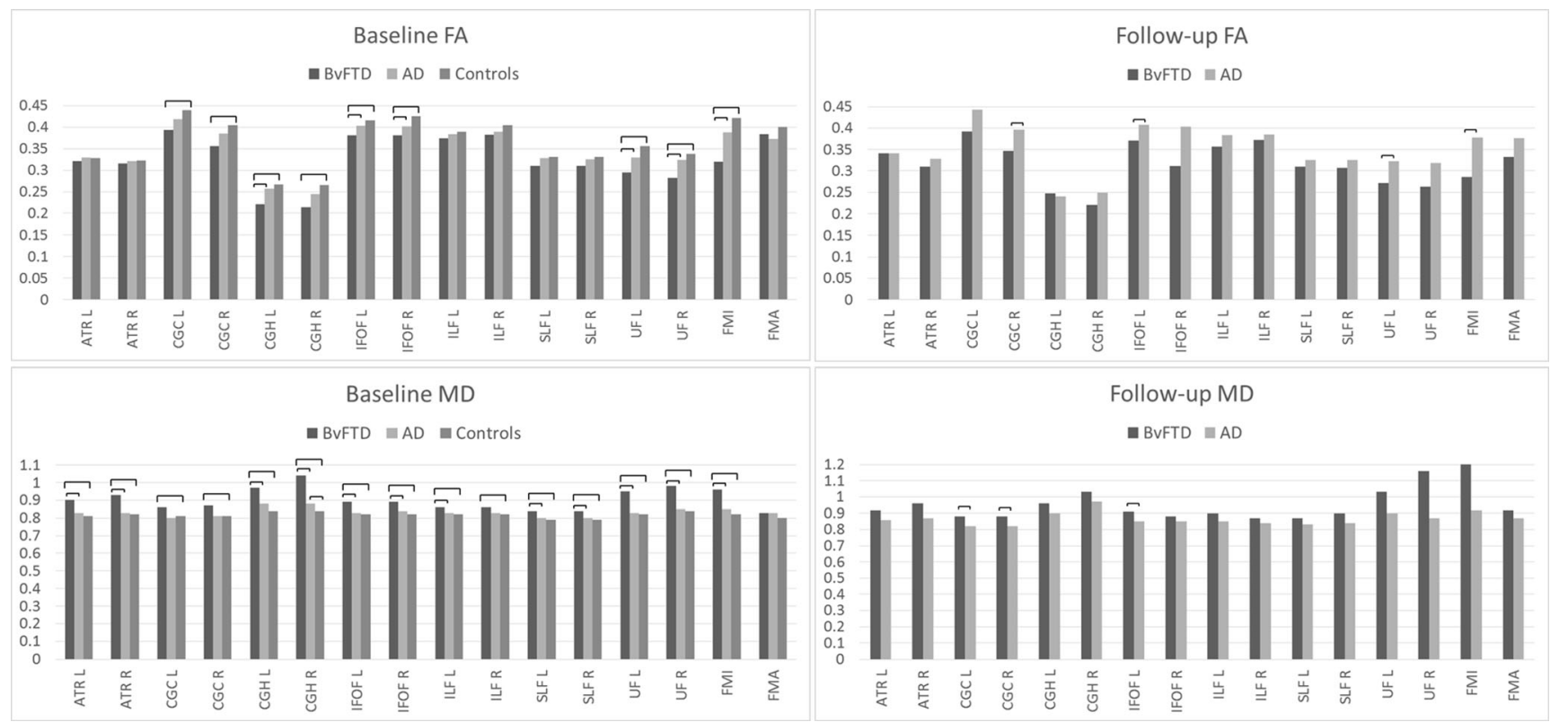

Fig. 1 Mean fractional anisotropy (FA) and mean diffusivity (MD) at baseline and follow-up for individual white matter tracts shown per group (Alzheimer's disease (AD), behavioural variant frontotemporal dementia (bvFTD) and controls). Significant differences $(p<0.05)$ between groups indicated by a horizontal line. CGH, cingulum (hippocampal); IFOF, inferior fronto-occipital fasciculus; ILF, inferior longitudinal fasciculus; SLF, superior longitudinal fasciculus; UF, uncinate fasciculus; FMI, forceps minor; FMA, forceps major; L, left; R, right 
additionally observed in the bilateral ATR, SLF and right CGH. Lower FA in bvFTD compared with AD was additionally observed in the left CGH. Further, bvFTD in comparison with $\mathrm{AD}$ showed higher $\mathrm{MD}$ and $\mathrm{RD}$ in the bilateral $\mathrm{CGH}$, higher MD and AxD in the left ILF and higher AxD in the right CGC. For an example of the affected WM tracts, see Fig. 2 where between-group differences in FA for individual WM tracts are shown.

\section{Functional connectivity}

Significant DMN within-group functional connectivity and between-group functional connectivity changes were not observed (Fig. 3).

\section{Follow-up (T1)}

\section{Participant characteristics}

Patients underwent a second MRI approximately 1 year later at T1 (mean 378 days). Six bvFTD patients did not undergo MRI at T1 and hence were excluded from T1 data analysis. Three of these patients had not been consented for a scan at $\mathrm{T} 1$ and three patients had progressed too severely. Data from 11 AD patients and 6 bvFTD patients were used for the analysis (Table 2). Participants did not differ in age $(t(15)=0.311$, $p>0.05)$ or gender $\left(\chi^{2}(1,15)=0.88, p>0.05\right)$.

\section{GM volume}

The total GM volume (\%ICV) was different between AD and bvFTD $(t(15)=-2.266, p<0.039)$ and was significantly lower in bvFTD $(0.27 \% \mathrm{ICV}, \mathrm{SD} 0.05)$ than in $\mathrm{AD}(0.32 \% \mathrm{ICV}, \mathrm{SD}$ $0.04)$.

\section{WM microstructure}

WM tracts were correctly identified in both groups, except for in one bvFTD and one AD patient where eight tracts could not be reconstructed due to low data quality. Data for these tracts were not used.

BvFTD in comparison with AD (Table 4, Figs. 1 and 2) showed lower FA and higher MD and RD in the right CGC and left IFOF. Additionally, higher MD in bvFTD compared with $\mathrm{AD}$ was observed in the left $\mathrm{CGC}$ and lower FA in the left UF and FMI. No differences were observed in $\mathrm{AxD}$.

The rate of change between $\mathrm{T} 1$ and $\mathrm{T} 0$ (Table 5) of FA in the cingulum was different between bvFTD and AD. Specifically, the rate of change of $\mathrm{FA}$ in the right $\mathrm{CGC}$ was higher in bvFTD versus a lower change in AD, whereas the rate of change of FA in the left $\mathrm{CGH}$ was lower in bvFTD versus a higher change in AD. Additionally, the rate of change of $\mathrm{AxD}$ in the right IFOF was also different between bvFTD and AD; namely, it was lower in bvFTD versus higher in $\mathrm{AD}$.

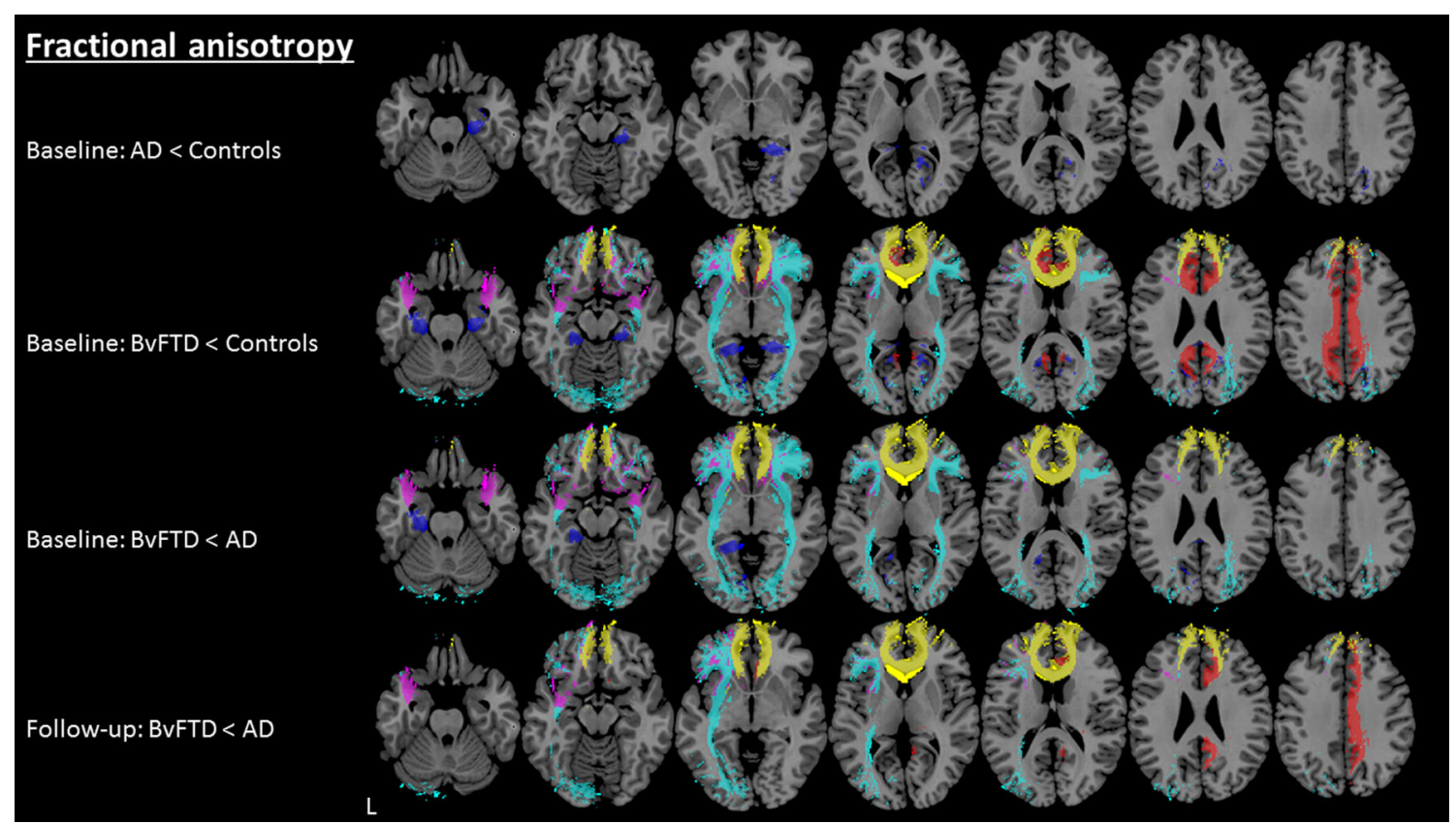

Fig. 2 Between-group fractional anisotropy differences $(p<0.05)$ in white matter tracts at baseline and follow-up. Light blue, inferior-fronto occipital fasciculus; yellow, forceps minor; red, cingulate cingulum; dark blue, hippocampal cingulum; pink, uncinate fasciculus; AD, Alzheimer's disease; bvFTD, behavioural variant frontotemporal dementia; L, left 
BvFTD

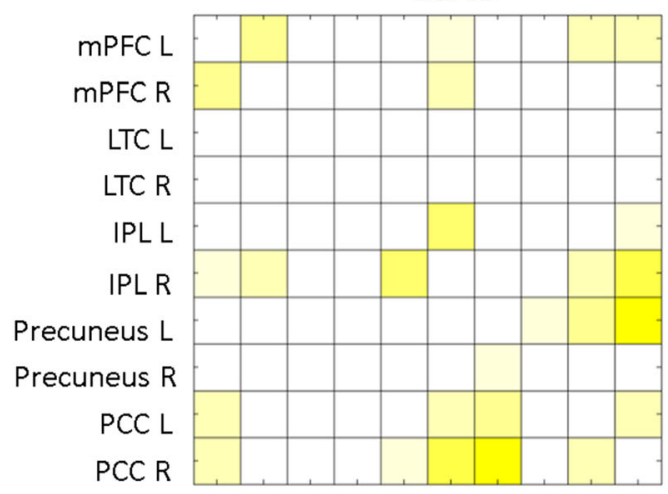

BvFTD vs AD

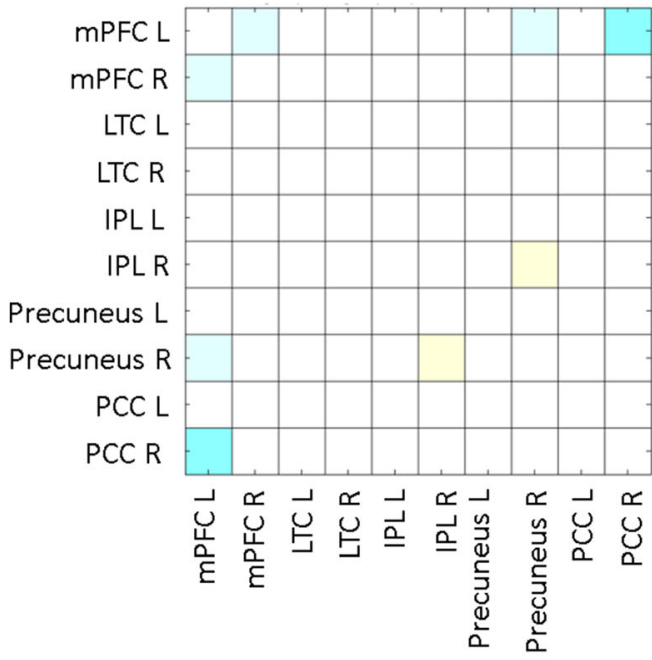

Fig. 3 Non-significant default mode network (DMN) connectivity $\left(p>0.05 ; \mathrm{FWE}_{\text {corrected }}\right)$ at baseline (T0). DMN functional connectivity for behavioural variant frontotemporal dementia (bvFTD), Alzheimer's disease $(\mathrm{AD})$ and controls is shown in row one. Between-group differences

\section{Functional connectivity}

Significant DMN within-group functional connectivity and between-group functional connectivity changes were not observed (Fig. 4). For this reason, added value and/or sensitivity of the rate of change analysis was not expected and therefore not performed.

\section{Discussion}

In this study, we explored group-wise quantitative measures of tract-specific WM microstructure and functional connectivity of the DMN to provide an initial indication of their diagnostic utility for early-stage and over time differentiation of AD and bvFTD. Quantitative tract-specific microstructural WM abnormalities, but not quantitative functional DMN connectivity, may aid early-stage - and possibly over time - differential diagnosis of bvFTD and AD. Microstructural WM abnormalities were
AD

Controls

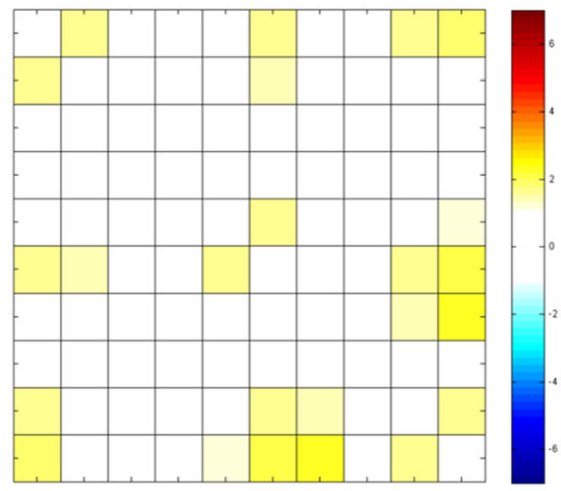

BvFTD vs controls

AD vs controls

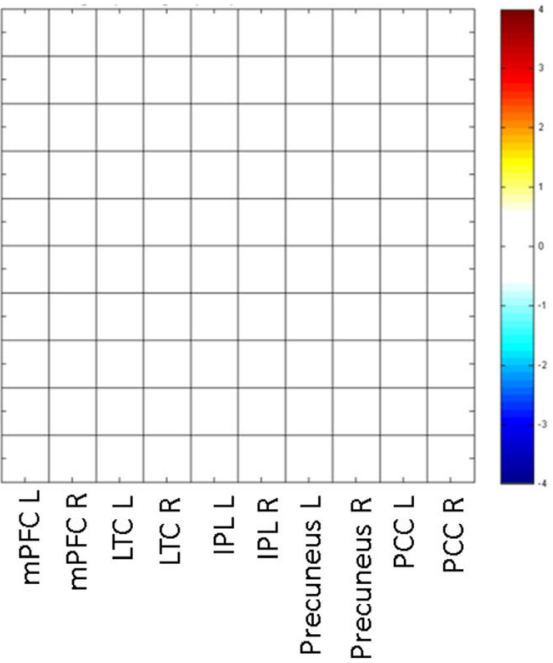

for DMN functional connectivity are shown in row two. Colours represent the $t$ values of between-region functional connectivity. mPFC, medial prefrontal cortex mPFC; LTC, lateral temporal cortex; IPL, inferior parietal lobule; PCC, posterior cingulate cortex; L, left; R, right

observed in widespread WM tracts in bvFTD, whereas they were only seen regionally in $\mathrm{AD}$. Additionally, at follow-up, the differences in tract-specific microstructural WM abnormalities between bvFTD and AD became less pronounced, although they were still stronger in bvFTD. Despite these diminished differences, the rate of change was very similar between bvFTD and $\mathrm{AD}$. It should be noted that this might be an underappreciation of differences due to bvFTD drop-out at follow-up.

\section{WM microstructure}

\section{Baseline}

Quantitative microstructural WM abnormalities were seen in bvFTD and AD in different WM tracts, suggesting a differential diagnostic role for assessing diffusion values in a clinical context. Tract-specific WM microstructural abnormalities were evident in bvFTD in all WM tracts investigated, but most pronounced in the FMI, CGH, CGC, IFOF and UF. These tracts 
Table 4 Mean FA, MD, RD and AxD for each WM tract, for bvFTD and $\mathrm{AD}$ at $\mathrm{T} 1$. The numbers shown are multiplied by a factor 1000

\begin{tabular}{|c|c|c|c|c|c|c|c|c|c|}
\hline \multirow[t]{2}{*}{ WM tract } & \multirow[t]{2}{*}{$\mathrm{L} / \mathrm{R}$} & \multicolumn{2}{|l|}{ FA } & \multicolumn{2}{|l|}{ MD } & \multicolumn{2}{|l|}{$\mathrm{RD}$} & \multicolumn{2}{|l|}{$\mathrm{AxD}$} \\
\hline & & BvFTD & $\mathrm{AD}$ & BvFTD & $\mathrm{AD}$ & BvFTD & $\mathrm{AD}$ & BvFTD & $\mathrm{AD}$ \\
\hline ATR & $\mathrm{L}$ & 341.44 & 341.57 & 0.92 & 0.86 & 0.74 & 0.69 & 1.33 & 1.24 \\
\hline ATR & $\mathrm{R}$ & 309.17 & 328.47 & 0.96 & 0.87 & 0.79 & 0.71 & 1.37 & 1.23 \\
\hline CGC & $\mathrm{L}$ & 392.49 & 443.31 & 0.88 & 0.82 & Excluded & Excluded & 1.28 & $1.22 *$ \\
\hline CGC & $\mathrm{R}$ & 346.15 & 396.49 & 0.88 & 0.82 & 0.71 & 0.63 & 1.24 & 1.19 \\
\hline $\mathrm{CGH}$ & $\mathrm{L}$ & 246.78 & 240.96 & 0.96 & 0.90 & 0.82 & 0.78 & 1.27 & 1.20 \\
\hline $\mathrm{CGH}$ & $\mathrm{R}$ & 220.07 & 248.85 & 1.03 & 0.97 & 0.91 & $0.81 *$ & 1.31 & 1.33 \\
\hline IFOF & $\mathrm{L}$ & 370.40 & 407.22 & 0.91 & 0.85 & 0.71 & 0.64 & 1.32 & $1.26^{*}$ \\
\hline IFOF & $\mathrm{R}$ & 311.60 & $403.42 *$ & 0.88 & 0.85 & 0.67 & 0.65 & 1.30 & 1.26 \\
\hline ILF & $\mathrm{L}$ & 357.26 & 383.46 & 0.90 & 0.85 & 0.71 & 0.65 & 1.28 & $1.23 *$ \\
\hline ILF & $\mathrm{R}$ & 371.54 & 384.99 & 0.87 & 0.84 & 0.68 & 0.66 & 1.27 & 1.23 \\
\hline SLF & $\mathrm{L}$ & 310.15 & 325.04 & 0.87 & 0.83 & 0.72 & 0.68 & 1.19 & 1.17 \\
\hline SLF & $\mathrm{R}$ & 306.60 & 325.68 & 0.90 & 0.84 & 0.75 & 0.68 & 1.26 & 1.18 \\
\hline UF & $\mathrm{L}$ & 271.33 & 323.24 & 1.03 & 0.90 & 0.87 & 0.73 & 1.35 & 1.25 \\
\hline UF & $\mathrm{R}$ & 262.51 & $317.80^{*}$ & 1.16 & $0.87^{*}$ & 1.00 & $0.71^{*}$ & 1.53 & $1.21 *$ \\
\hline FMI & $\mathrm{n} / \mathrm{a}$ & 285.17 & 378.20 & 1.20 & 0.92 & 1.02 & 0.71 & 1.54 & 1.33 \\
\hline FMA & $\mathrm{n} / \mathrm{a}$ & 331.97 & 376.31 & 0.92 & 0.87 & 0.74 & 0.66 & 1.36 & 1.35 \\
\hline
\end{tabular}

Italicised entries $=$ vs other patient groups $p<0.05$

$F A$, fractional anisotropy; $M D$, mean diffusivity; $R D$, radial diffusivity; $A x D$, axial diffusivity; $W M$, white matter; $b v F T D$, behavioural variant frontotemporal dementia; $A D$, Alzheimer's disease; ATR, anterior thalamic radiation; $C G C$, cingulum (cingulate); $C G H$, cingulum (hippocampal); $I F O F$, inferior fronto-occipital fasciculus; $I L F$, inferior longitudinal fasciculus; $S L F$, superior longitudinal fasciculus; $U F$, uncinate fasciculus; $F M I$, forceps minor; FMA, forceps major; $L$, left; $R$, right

*Unequal variance. Significance tested with Welch-ANOVA have been associated with cognitive domains characteristically affected in bvFTD [3]: the FMI with disinhibition and executive functioning [23, 32], CGH with memory and executive functioning [33, 34], CGC with cognitive control [35], IFOF with social cognition and emotional functioning $[36,37]$ and the UF with apathy, disinhibition and behavioural dyscontrol $[23,38,39]$. Unsurprisingly, WM abnormalities were not observed in the FMA, which is a posterior tract associated with visuospatial functioning [40], a domain generally preserved in bvFTD [41]. Microstructural WM abnormalities in bvFTD were evident in comparison with both $\mathrm{AD}$ and controls, but even more pronounced in comparison with the latter. This smaller difference between bvFTD and AD could indicate that changes in the $\mathrm{WM}$ in $\mathrm{AD}$ were already ongoing. $\mathrm{AD}$ in comparison with controls only showed microstructural abnormalities at baseline, specifically only in the $\mathrm{CGH}$, suggesting the importance of this structure in AD. Previously, the CGH has been associated with memory functioning $[42,43]$, which is characteristically impaired in $\mathrm{AD}[2]$.

\section{Follow-up}

At follow-up, microstructural WM abnormalities were still more pronounced in bvFTD than in $\mathrm{AD}$, but in fewer tracts, specifically in the left IFOF and UF and right CGC and FMI, suggesting these may be important for differentiating between bvFTD and AD at later stages. However, some caution is warranted, as six out of the twelve bvFTD patients did not return for follow-up. If these patients were more advanced than patients who participated at follow-up, not including them may have led to underappreciating WM abnormalities in bvFTD.

CGC involvement in bvFTD is in line with previous literature showing classification of bvFTD and controls to be best achieved using FA in the cingulum bundle [44]. The IFOF has been previously associated with a variety of cognitive domains, such as emotion recognition [37], executive functioning $[34,45]$ and processing speed [46], of which many have been associated with bvFTD $[22,47,48]$. The UF and FMI are both known to be important in bvFTD and are associated with characteristic bvFTD symptoms [23, 32, 38].

The rate of change, in terms of the difference in diffusivity abnormalities between baseline and follow-up, showed a faster decline in WM microstructure of the right CGC in bvFTD and the left $\mathrm{CGH}$ and right IFOF in $\mathrm{AD}$. In line with the observed baseline and follow-up changes and previous literature [44], this may suggest a differential involvement of the cingulum, in which the anterior part is more affected in bvFTD and the posterior part in AD. This is supported by the 
Table 5 Mean difference score (T1 minus T0) of FA, MD, RD and $\mathrm{AxD}$ for each WM tract, for bvFTD and AD. The numbers shown are multiplied by a factor 1000

\begin{tabular}{|c|c|c|c|c|c|c|c|c|c|}
\hline \multirow[t]{2}{*}{ WM tract } & \multirow[t]{2}{*}{$\mathrm{L} / \mathrm{R}$} & \multicolumn{2}{|l|}{ FA } & \multicolumn{2}{|l|}{ MD } & \multicolumn{2}{|l|}{$\mathrm{RD}$} & \multicolumn{2}{|l|}{$\mathrm{AxD}$} \\
\hline & & BvFTD & $\mathrm{AD}$ & BvFTD & $\mathrm{AD}$ & BvFTD & $\mathrm{AD}$ & BvFTD & $\mathrm{AD}$ \\
\hline ATR & L & 9.80 & 8.75 & 0.03 & 0.03 & 0.02 & 0.02 & 0.05 & 0.05 \\
\hline ATR & $\mathrm{R}$ & -9.84 & 5.81 & 0.02 & 0.04 & 0.03 & 0.02 & 0.03 & 0.06 \\
\hline CGC & $\mathrm{L}$ & -5.59 & 20.23 & 0.01 & 0.01 & 0.01 & 0.0003 & 0.002 & 0.01 \\
\hline CGC & $\mathrm{R}$ & -29.77 & 10.44 & 0.02 & 0.01 & 0.03 & 0.001 & -0.002 & $0.01 *$ \\
\hline $\mathrm{CGH}$ & $\mathrm{L}$ & 13.84 & -19.95 & 0.03 & 0.01 & 0.02 & 0.01 & 0.04 & -0.003 \\
\hline $\mathrm{CGH}$ & $\mathrm{R}$ & -5.78 & -1.84 & 0.03 & 0.08 & 0.04 & 0.05 & 0.02 & 0.14 \\
\hline IFOF & $\mathrm{L}$ & -5.42 & -3.17 & 0.01 & 0.01 & 0.02 & 0.01 & 0.02 & 0.02 \\
\hline IFOF & $\mathrm{R}$ & -73.01 & $-4.87 *$ & -0.003 & 0.01 & -0.01 & 0.01 & -0.008 & 0.02 \\
\hline ILF & $\mathrm{L}$ & -12.86 & -1.62 & 0.03 & $0.01 *$ & 0.03 & 0.01 & 0.04 & $0.02 *$ \\
\hline ILF & $\mathrm{R}$ & -13.69 & -8.00 & 0.01 & 0.01 & 0.02 & 0.01 & 0.01 & 0.01 \\
\hline SLF & $\mathrm{L}$ & 1.28 & -3.97 & 0.03 & 0.03 & 0.03 & 0.03 & 0.04 & 0.05 \\
\hline SLF & $\mathrm{R}$ & 6.20 & -2.72 & 0.04 & 0.05 & 0.02 & 0.04 & 0.07 & 0.06 \\
\hline UF & $\mathrm{L}$ & -25.28 & -12.54 & 0.09 & 0.07 & 0.10 & 0.06 & 0.08 & 0.07 \\
\hline UF & $\mathrm{R}$ & -13.80 & -10.44 & 0.15 & $0.02 *$ & 0.15 & $0.02 *$ & 0.17 & $0.02 *$ \\
\hline FMI & $\mathrm{n} / \mathrm{a}$ & -30.22 & -13.49 & 0.23 & 0.07 & 0.23 & 0.06 & 0.24 & 0.10 \\
\hline FMA & $\mathrm{n} / \mathrm{a}$ & -37.71 & $-3.78^{*}$ & 0.08 & 0.04 & 0.09 & $0.03 *$ & 0.04 & 0.06 \\
\hline
\end{tabular}

Italicised entries $=$ vs other patient group $p<0.05$

$F A$, fractional anisotropy; $M D$, mean diffusivity; $R D$, radial diffusivity; $A x D$, axial diffusivity; $W M$, white matter; $b v F T D$, behavioural variant frontotemporal dementia; $A D$, Alzheimer's disease; ATR, anterior thalamic radiation; $C G C$, cingulum (cingulate); $C G H$, cingulum (hippocampal); $I F O F$, inferior fronto-occipital fasciculus; $I L F$, inferior longitudinal fasciculus; $S L F$, superior longitudinal fasciculus; $U F$, uncinate fasciculus; $F M I$, forceps minor; FMA, forceps major; $L$, left; $R$, right

*Unequal variance, significance tested with Welch-ANOVA macrostructural frontotemporal (anterior) and temporoparietal (posterior) involvement in, respectively, bvFTD and $\mathrm{AD}[40$, 49]. The IFOF has been associated with many different cognitive functions - as described above — but has as yet not been specifically linked to AD or bvFTD. However, the observed left versus right IFOF involvement in, respectively,

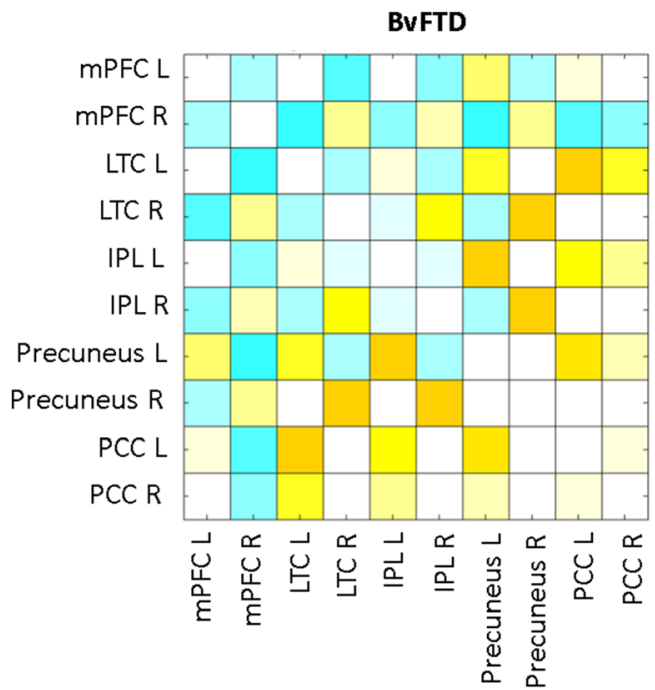

Fig. 4 Non-significant default mode network (DMN) connectivity $\left(p>0.05 ; \mathrm{FWE}_{\text {corrected }}\right)$ at follow-up (T1). DMN functional connectivity for behavioural variant frontotemporal dementia (bvFTD) and Alzheimer's disease (AD) is shown in columns one and two, respectively. Between-group comparison for DMN functional connectivity is shown in
AD
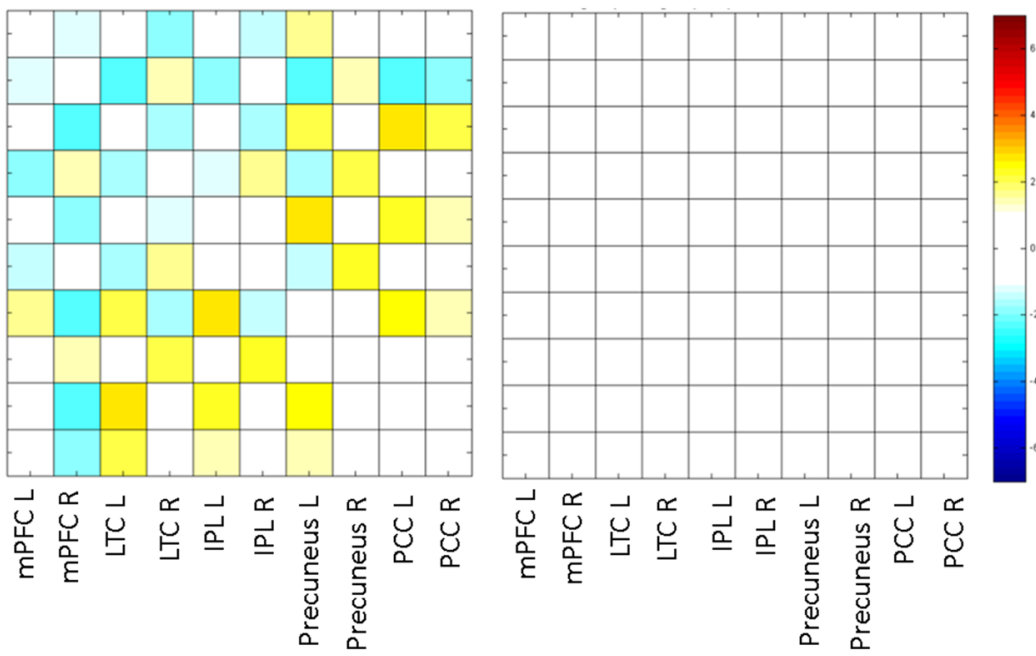

column three. Colours represent the $t$ values of between-region functional connectivity. mPFC, medial prefrontal cortex mPFC; LTC, lateral temporal cortex; IPL, inferior parietal lobule; PCC, posterior cingulate cortex; $\mathrm{L}$, left; R, right 
bvFTD and AD suggests that a disease-specific link may in fact be present.

\section{Diffusion metrics sensitive to group differences}

Differences between AD and bvFTD were most pronounced in MD and RD at baseline, and in FA (and to a lesser extent, $\mathrm{MD}$ and RD) at follow-up. First, this suggests that myelin abnormalities are more pronounced in bvFTD, as RD is thought to represent myelin damage [50] and $\mathrm{AxD}$ axonal loss [51]. As MD and FA are a combination of these measures, it may be that their changes observed here are induced by the changes in $\mathrm{RD}$ rather than $\mathrm{AxD}$. Second, this suggests-also in line with previous literature $[16,44]$ - that FA, MD and RD are most sensitive to group changes and are therefore recommended for differentiation between $\mathrm{AD}$ and bvFTD.

\section{Functional connectivity}

Functional DMN connectivity between AD and bvFTD was not different using our quantitative method. Previous literature observed differences in DMN regions using whole-brain independent component analysis [18, 52-54], such as increased parietal DMN connectivity in bvFTD and decreased parietal DMN connectivity in AD. However, in this study, we aimed to assess a different approach that may be used clinically, i.e. a quantitative measure of functional connectivity between DMN regions. The small sample size of this study, and thus low power, may have left possible group effects undetected. However, clinical use warrants sensitivity of measures at an individual patient level; hence, a low sensitivity of quantitative functional DMN connectivity does not seem suitable for individual diagnostics.

\section{Limitations}

This study knows some limitations. First, the small sample size limits interpretation and generalizability of the results and it may particularly lead to underestimation of between-group differences. However, the findings of this study are in line with the literature and may indicate clinical utility of DTI, but not rsfMRI, on an individual patient level. Second, sample size was smaller at follow-up than at baseline which may have induced an underappreciation of abnormality severity at follow-up and rate of change differences in the bvFTD patients.

\section{Conclusion}

In this explorative group-wise study of quantitative brain MR measures in dementia, we aimed to provide an indication of their usefulness for differentiation between AD and bvFTD at multiple time points. We observed that quantitative tractspecific microstructural WM abnormalities, but not quantitative functional connectivity of the DMN, may aid differential diagnosis of bvFTD and AD at the early-stage and possibly over time. Specifically, pronounced microstructural WM changes in anterior WM tracts may differentiate bvFTD from $\mathrm{AD}$, and microstructural WM abnormalities of the hippocampal cingulum, in the absence of other microstructural WM changes, may differentiate AD from bvFTD.

Acknowledgements We want to thank Dr Marjolein Verly for sharing her functional connectivity insights.

Funding This project was financially supported by a personal fellowship granted by the Erasmus University Rotterdam.

\section{Compliance with ethical standards}

Guarantor The scientific guarantor of this publication is Dr M. Smits.

Conflict of interest The authors of this manuscript declare no relationships with any companies, whose products or services may be related to the subject matter of the article.

Statistics and biometry One of the authors has significant statistical expertise.

Informed consent Written informed consent was obtained from all subjects (patients) in this study.

Ethical approval Institutional Review Board approval was obtained.

Study subjects or cohorts overlap Some study subjects or cohorts have been previously reported in:

Meijboom R, Steketee RM, Ham LS, van der Lugt A, van Swieten JC, Smits M. Hemispheric dissociation of microstructural white matter and functional connectivity abnormalities in semantic and behavioural variant frontotemporal dementia. J Alzheimers Dis. 2017;56(2):789-804. doi: 10.3233/JAD-160564.

Meijboom R, Steketee RM, de Koning I, Osse RJ, Jiskoot LC, de Jong FJ, van der Lugt A, van Swieten JC, Smits M. Functional connectivity and microstructural white matter changes in phenocopy frontotemporal dementia. Eur Radiol. 2017 Apr;27(4):1352-1360. doi: 10.1007/s00330016-4490-4.

Steketee RM*, Meijboom R*, De Groot M, Bron EE, Niessen WJ, Van der Lugt A, Van Swieten JC, Smits M. Concurrent white and grey matter degeneration of disease-specific networks in early-stage Alzheimer's disease and behavioural variant frontotemporal dementia. Neurobiology of Aging. 2016 May; 119-128. doi: 10.1016/j.neurobiolaging.2016.03.03.1. * These authors contributed equally to this work.

Bron EE, Smits M, Papma JM, Steketee RM, Meijboom R, De Groot M, Van Swieten JC, Niessen WJ, Klein S. Multiparametric computeraided differential diagnosis of Alzheimer's disease and frontotemporal dementia using structural and advanced MRI. Eur Radiol. 2017 Aug; 27(8):3372-3382. doi: 10.1007/s00330-016-4691-x.

Steketee RM, Meijboom R, Bron EE, Osse RJ, de Koning I, Jiskoot LC, Klein S, de Jong FJ, van der Lugt A, van Swieten JC, Smits M. Structural and functional brain abnormalities place phenocopy frontotemporal dementia (FTD) in the FTD spectrum. Neuroimage Clinical. 2016 Apr ; 11:595-605.

Steketee RM, Bron EE, Meijboom R, Houston GC, Klein S, Mutsaerts HJ, Mendez Orellana CP, de Jong FJ, van Swieten JC, van 
der Lugt A, Smits M. Early-stage differentiation between presenile Alzheimer's disease and frontotemporal dementia using arterial spin labeling MRI. Eur Radiol. 2016 Jan;26(1):244-53. doi: 10.1007/s00330015-3789-x.

Bron EE, Smits M, Van der Flier WM, Vrenken H, Barkhof F, Scheltens P, Papma JM, Steketee RM, Méndez Orellana C, Meijboom R et al Standardized evaluation of algorithms for computer-aided diagnosis of dementia based on structural MRI: the CADDementia challenge. Neuroimage. 2015 May 1;111:562-79. doi: 10.1016/j.neuroimage.2015.01.048.

Bron EE, Steketee RM, Houston GC, Oliver RA, Achterberg HC, Loog M, van Swieten JC, Hammers A, Niessen WJ, Smits M, Klein S; Alzheimer's Disease Neuroimaging Initiative. Diagnostic classification of arterial spin labeling and structural MRI in presenile early stage dementia. Human Brain Mapping. 2014 Sep;35(9):4916-31. doi: $10.1002 / \mathrm{hbm} .22522$

\section{Methodology \\ - Prospective \\ - Case-control study \\ - Performed at one institution}

Open Access This article is distributed under the terms of the Creative Commons Attribution 4.0 International License (http:// creativecommons.org/licenses/by/4.0/), which permits unrestricted use, distribution, and reproduction in any medium, provided you give appropriate credit to the original author(s) and the source, provide a link to the Creative Commons license, and indicate if changes were made.

Publisher's note Springer Nature remains neutral with regard to jurisdictional claims in published maps and institutional affiliations.

\section{References}

1. Greicius MD, Geschwind MD, Miller BL (2002) Presenile dementia syndromes: an update on taxonomy and diagnosis. J Neurol Neurosurg Psychiatry 72:691-700

2. McKhann GM, Knopman DS, Chertkow H et al (2011) The diagnosis of dementia due to Alzheimer's disease: recommendations from the National Institute on Aging-Alzheimer's Association workgroups on diagnostic guidelines for Alzheimer's disease. Alzheimers Dement 7:263-269

3. Rascovsky K, Hodges JR, Knopman D et al (2011) Sensitivity of revised diagnostic criteria for the behavioural variant of frontotemporal dementia. Brain 134:2456-2477

4. Neary D, Snowden JS, Northen B, Goulding P (1988) Dementia of frontal lobe type. J Neurol Neurosurg Psychiatry 51:353-361

5. Bathgate D, Snowden JS, Varma A, Blackshaw A, Neary D (2001) Behaviour in frontotemporal dementia, Alzheimer's disease and vascular dementia. Acta Neurol Scand 103:367-378

6. Hallam BJ, Silverberg ND, Lamarre AK, Mackenzie IR, Feldman HH (2007) Clinical presentation of prodromal frontotemporal dementia. Am J Alzheimers Dis Other Demen 22:456-467

7. Varma AR, Snowden JS, Lloyd JJ, Talbot PR, Mann DM, Neary D (1999) Evaluation of the NINCDS-ADRDA criteria in the differentiation of Alzheimer's disease and frontotemporal dementia. J Neurol Neurosurg Psychiatry 66:184-188

8. Jenner C, Reali G, Puopolo M, Silveri MC (2006) Can cognitive and behavioural disorders differentiate frontal variantfrontotemporal dementia from Alzheimer's disease at early stages? Behav Neurol 17:89-95

9. Gregory CA, Serra-Mestres J, Hodges JR (1999) Early diagnosis of the frontal variant of frontotemporal dementia: how sensitive are standard neuroimaging and neuropsychologic tests? Neuropsychiatry Neuropsychol Behav Neurol 12:128-135

10. Kipps CM, Davies RR, Mitchell J, Kril JJ, Halliday GM, Hodges JR (2007) Clinical significance of lobar atrophy in frontotemporal dementia: application of an MRI visual rating scale. Dement Geriatr Cogn Disord 23:334-342

11. Rosso SM, Heutink P, Tibben A, Niermeijer MF, van Swieten JC (2000) New insights in frontotemporal dementia. Ned Tijdschr Geneeskd 144:1575-1580

12. Sperling RA, Aisen PS, Beckett LA et al (2011) Toward defining the preclinical stages of Alzheimer's disease: recommendations from the National Institute on Aging-Alzheimer's Association workgroups on diagnostic guidelines for Alzheimer's disease. Alzheimers Dement 7:280-292

13. Zhang Y, Schuff N, Ching C et al (2011) Joint assessment of structural, perfusion, and diffusion MRI in Alzheimer's disease and frontotemporal dementia. Int J Alzheimers Dis. https://doi.org/10. 4061/2011/546871

14. Zhang Y, Schuff N, Du AT et al (2009) White matter damage in frontotemporal dementia and Alzheimer's disease measured by diffusion MRI. Brain 132:2579-2592

15. Daianu M, Mendez MF, Baboyan VG et al (2015) An advanced white matter tract analysis in frontotemporal dementia and earlyonset Alzheimer's disease. Brain Imaging Behav 10:1038-1053

16. Mahoney CJ, Ridgway GR, Malone IB et al (2014) Profiles of white matter tract pathology in frontotemporal dementia. Hum Brain Mapp 35:4163-4179

17. Lu PH, Lee GJ, Shapira J et al (2014) Regional differences in white matter breakdown between frontotemporal dementia and earlyonset Alzheimer's disease. J Alzheimers Dis 39:261-269

18. Zhou J, Greicius MD, Gennatas ED et al (2010) Divergent network connectivity changes in behavioural variant frontotemporal dementia and Alzheimer's disease. Brain 133:1352-1367

19. Folstein MF, Folstein SE, McHugh PR (1975) "Mini-mental state". A practical method for grading the cognitive state of patients for the clinician. J Psychiatr Res 12:189-198

20. Bron EE, Steketee RM, Houston GC et al (2014) Diagnostic classification of arterial spin labeling and structural MRI in presenile early stage dementia. Hum Brain Mapp 35:4916-4931

21. Duering M, Gonik M, Malik R et al (2013) Identification of a strategic brain network underlying processing speed deficits in vascular cognitive impairment. Neuroimage 66:177-183

22. Tartaglia MC, Zhang Y, Racine C et al (2012) Executive dysfunction in frontotemporal dementia is related to abnormalities in frontal white matter tracts. J Neurol 259:1071-1080

23. Hornberger M, Geng J, Hodges JR (2011) Convergent grey and white matter evidence of orbitofrontal cortex changes related to disinhibition in behavioural variant frontotemporal dementia. Brain 134:2502-2512

24. Epstein KA, Cullen KR, Mueller BA, Robinson P, Lee S, Kumra S (2014) White matter abnormalities and cognitive impairment in early-onset schizophrenia-spectrum disorders. J Am Acad Child Adolesc Psychiatry 53:362-372

25. Mike A, Strammer E, Aradi M et al (2013) Disconnection mechanism and regional cortical atrophy contribute to impaired processing of facial expressions and theory of mind in multiple sclerosis: a structural MRI study. PLoS One. https://doi.org/10.1371/journal. pone.0082422

26. Borroni B, Brambati SM, Agosti C et al (2007) Evidence of white matter changes on diffusion tensor imaging in frontotemporal dementia. Arch Neurol 64:246-251

27. Sarubbo S, De Benedictis A, Merler S et al (2015) Towards a functional atlas of human white matter. Hum Brain Mapp 36: $3117-3136$

28. Jenkinson M, Beckmann CF, Behrens TE, Woolrich MW, Smith SM (2012) FSL. Neuroimage 62:782-790 
29. Hammers A, Allom R, Koepp MJ et al (2003) Three-dimensional maximum probability atlas of the human brain, with particular reference to the temporal lobe. Hum Brain Mapp 19:224-247

30. Ebisch SJH, Gallese V, Willems RM et al (2011) Altered intrinsic functional connectivity of anterior and posterior insula regions in high-functioning participants with autism spectrum disorder. Hum Brain Mapp 32:1013-1028

31. Verly M, Verhoeven J, Zink I et al (2014) Altered functional connectivity of the language network in ASD: Role of classical language areas and cerebellum. Neuroimage Clin 4:374-382

32. Pérez-Iglesias R, Tordesillas-Gutiérrez D, McGuire PK et al (2010) White Matter Integrity and Cognitive Impairment in First-Episode Psychosis. Am J Psychiatry 167:451-458

33. Irish M, Devenney E, Wong S et al (2013) Neural substrates of episodic memory dysfunction in behavioural variant frontotemporal dementia with and without C9ORF72 expansions. Neuroimage Clin 2:836-843

34. Santiago C, Herrmann N, Swardfager W et al (2015) White matter microstructural integrity is associated with executive function and processing speed in older adults with coronary artery disease. Am J Geriatr Psychiatry 23:754-763

35. Metzler-Baddeley C, Jones DK, Steventon J, Westacott L, Aggleton JP, O'Sullivan MJ (2012) Cingulum microstructure predicts cognitive control in older age and mild cognitive impairment. J Neurosci 32:17612-17619

36. Jalbrzikowski M, Villalon-Reina JE, Karlsgodt KH et al (2014) Altered white matter microstructure is associated with social cognition and psychotic symptoms in 22q11.2 microdeletion syndrome. Front Behav Neurosci. https://doi.org/10.3389/fnbeh.2014. 00393

37. Crespi C, Cerami C, Dodich A et al (2014) Microstructural white matter correlates of emotion recognition impairment in amyotrophic lateral sclerosis. Cortex 53:1-8

38. Powers JP, Massimo L, McMillan CT et al (2014) White matter disease contributes to apathy and disinhibition in behavioral variant frontotemporal dementia. Cogn Behav Neurol 27:206-214

39. Whitwell JL, Avula R, Senjem ML et al (2010) Gray and white matter water diffusion in the syndromic variants of frontotemporal dementia. Neurology 74:1279-1287

40. Braak H, Braak E (1991) Neuropathological stageing of Alzheimerrelated changes. Acta Neuropathol 82:239-259

41. Laforce R Jr (2013) Behavioral and language variants of frontotemporal dementia: a review of key symptoms. Clin Neurol Neurosurg 115:2405-2410
42. Bozzali M, Giulietti G, Basile B et al (2012) Damage to the cingulum contributes to Alzheimer's disease pathophysiology by deafferentation mechanism. Hum Brain Mapp 33:1295-1308

43. Irish M, Hornberger M, El Wahsh S et al (2014) Grey and white matter correlates of recent and remote autobiographical memory retrieval-insights from the dementias. PLoS One. https://doi.org/ 10.1371/journal.pone.0113081

44. Mahoney CJ, Simpson IJA, Nicholas JM et al (2015) Longitudinal diffusion tensor imaging in frontotemporal dementia. Ann Neurol 77:33-46

45. Sun X, Liang Y, Wang J et al (2014) Early frontal structural and functional changes in mild white matter lesions relevant to cognitive decline. J Alzheimers Dis 40:123-134

46. Liu X, Lai Y, Wang X et al (2013) Reduced white matter integrity and cognitive deficit in never-medicated chronic schizophrenia: a diffusion tensor study using TBSS. Behav Brain Res 252:157-163

47. Balconi M, Cotelli M, Brambilla M et al (2015) Understanding emotions in frontotemporal dementia: the explicit and implicit emotional cue mismatch. J Alzheimers Dis 46:211-225

48. Block NR, Sha SJ, Karydas AM et al (2016) Frontotemporal dementia and psychiatric illness: emerging clinical and biological links in gene carriers. Am J Geriatr Psychiatry 24:107-116

49. Broe M, Hodges JR, Schofield E, Shepherd CE, Kril JJ, Halliday GM (2003) Staging disease severity in pathologically confirmed cases of frontotemporal dementia. Neurology 60:1005-1011

50. Song SK, Sun SW, Ramsbottom MJ, Chang C, Russell J, Cross AH (2002) Dysmyelination revealed through MRI as increased radial (but unchanged axial) diffusion of water. Neuroimage 17:14291436

51. Song SK, Sun SW, Ju WK, Lin SJ, Cross AH, Neufeld AH (2003) Diffusion tensor imaging detects and differentiates axon and myelin degeneration in mouse optic nerve after retinal ischemia. Neuroimage 20:1714-1722

52. Hafkemeijer A, Möller C, Dopper EGP et al (2016) Differences in structural covariance brain networks between behavioral variant frontotemporal dementia and Alzheimer's disease. Hum Brain Mapp 37:978-988

53. Hafkemeijer A, Möller C, Dopper EGP et al (2015) Resting state functional connectivity differences between behavioral variant frontotemporal dementia and Alzheimer's disease. Front Hum Neurosci. https://doi.org/10.3389/fnhum.2015.00474

54. Filippi M, Agosta F, Scola E et al (2013) Functional network connectivity in the behavioral variant of frontotemporal dementia. Cortex 49:2389-2401 